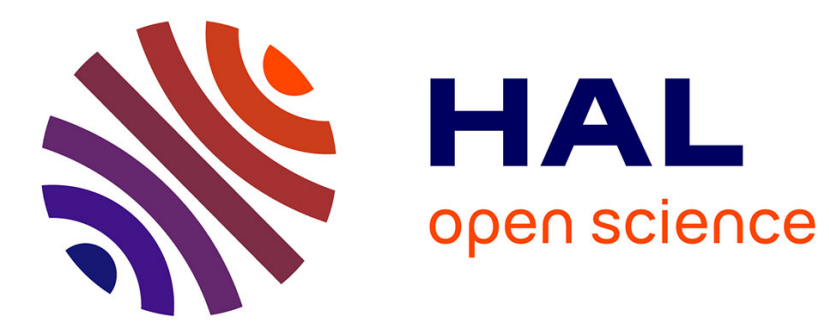

\title{
State Observer and Observability Conditions for a Class of Hybrid Continuous-Discrete Dynamic System
}

Jean-Pierre Barbot, Mohamed Djemai, Noureddine Manamanni

\section{To cite this version:}

Jean-Pierre Barbot, Mohamed Djemai, Noureddine Manamanni. State Observer and Observability Conditions for a Class of Hybrid Continuous-Discrete Dynamic System. CDC 2007, Dec 2007, New Orleans, United States. inria-00177803

\section{HAL Id: inria-00177803 \\ https://hal.inria.fr/inria-00177803}

Submitted on 9 Oct 2007

HAL is a multi-disciplinary open access archive for the deposit and dissemination of scientific research documents, whether they are published or not. The documents may come from teaching and research institutions in France or abroad, or from public or private research centers.
L'archive ouverte pluridisciplinaire HAL, est destinée au dépôt et à la diffusion de documents scientifiques de niveau recherche, publiés ou non, émanant des établissements d'enseignement et de recherche français ou étrangers, des laboratoires publics ou privés. 


\title{
State Observer and Observability Conditions for a Class of Hybrid Continuous-Discrete Dynamic System
}

\author{
J.P. BARBOT, M. DJEMAI and N. MANAMANNI, *
}

October 10, 2007

\begin{abstract}
This paper deals with observability conditions and state observer design for a class of hybrid systems combining continuous and discrete dynamics. The main contribution of the work lies in the performed observability conditions for this class of systems and a design of hybrid observer to reconstruct both continuous and discrete states starting only from the knowledge of a continuous output. An illustrative example is presented showing the efficiency of the proposed observer.
\end{abstract}

\section{Introduction}

Hybrid dynamical systems (HDS) was widely studied in literature these last decades in science and engineering, (see for example [1]). A common definition is that they are modelled or defined as a combination of both continuous and discrete event subsystems. This paper addresses observability conditions and state observer design for a particular class of hybrid systems with combined continuous and discrete state dynamics and called hybrid systems in state. Such models can represent a very broad range of systems for which the control and/or observer design problems remain still open. Indeed, some systems may be controlled by discrete system when computers are used in the control procedure. Also, in some applications in electrical

${ }^{*}$ M. Djemaï and J.P. Barbot (IEEE Member, and Member of Projet Alien INRIAFuturs) are with ECS, ENSEA, 6 avenue du Ponceau, 95014 Cergy, FRANCE \{djemai, barbot\}@ensea.fr. N. Manamanni (IEEE Member) is with CReSTIC, University of Reims, Moulin de la Housse BP 1039, 51687 REIMS cedex 2 - FRANCE, noureddine.manamanni@univ-reims.fr 
drive, this type of representation can be met after discretization of a part of the dynamics which will be controlled by a discrete system (system with commutation). In the case of the electric motors, the use of the PWM (Plush Width Modulation) is generally used. The PWM being discrete by definition, since the system works by commutation and makes it possible to control the electrical part of the engine (see for example [9, 22]). In this case, the control strategy consists in discretizing the electrical part of the dynamics (which will be controlled by the orders of commutations) and to keep the mechanical part in continuous time. Note that, in the literature, several papers have defined observability for different classes of hybrid systems (42, 3, 4, 5, 7, 8, 20, 21]). This paper concerns hybrid observer design for the following particular class of HDS:

$$
\begin{array}{rc}
\dot{x} & =F(x, Z(k)) \\
Z(k+1) & =A_{d} Z(k)+\beta_{d}(v(k), y(k)) \\
y & =y_{c}=H(x)=\left[h_{1}(x), \ldots, h_{p}(x)\right]
\end{array}
$$

where $x \in \mathcal{N}$, with $\mathcal{N}$ an open set of $\Re^{n}$, is the continuous state vector, $Z=\left(z_{1}, \ldots, z_{m}\right)^{T} \in \mathcal{M}$ with $\mathcal{M}$ an open set of $\Re^{m}$, is the discrete state vector with $Z(t)=Z\left(t_{k}\right)=Z(k)$ for $t \in\left[t_{k}, t_{k+1}\left[\forall k>0 . A_{d} \in \Re^{m \times m}\right.\right.$, is constant matrix with appropriate dimension and $F(x, Z(k)), H(x)$ and $\beta_{d}$ are a vectors functions of appropriate dimensions, $y(k)=y\left(t=t_{k}\right)$ is the output and $v(k) \in \Re^{\nu}$ a known input.

This work deals with the hybrid observer design in the case where the continuous output subsystem is available and all the switching instants $t_{k}$ i.e. the moments when the discrete system switches, are also available and supposed to be known throughout this paper. Nevertheless, it is possible to overcome the previous assumption by using some technics (see for example ([12])).

REMARK 1 The considered class of $\boldsymbol{H D S}$ is supposed without jumps for the continuous subsystem i.e., $x\left(t_{k}^{+}\right)=x\left(t_{k}^{-}\right)$where $t_{k}$ is the time when the discrete state switches from $Z(k-1)$ to $Z(k)$ and $t_{k}^{+}$and $t_{k}^{-}$are the instants just before and just after the switching time respectively.

\section{Hybrid observability conditions}

Throughout this paper, one considers a class of HDS of form (11) combining continuous and discrete dynamics and supposed to be bounded for both continuous and discrete states and verifies the following assumptions: 
Assumption 1 The period $T_{k}=t_{k+1}-t_{k}$ for the dynamics (9) is supposed to check the following condition:

$$
T_{k}>\tau_{m}>0 \quad \forall k \geq 0
$$

This in order to avoid the particular problem of systems with Zeno phenomena.

Let us now introduce some observability definitions for the considered subsystems of (11).

Definition 1 A pair of points $x^{0}$ and $x^{1}$ in $\mathcal{N}$ are uniformly distinguishable if for all input sequences $Z(k) \forall k \geq 0$, generated by (2), the system (1) generates solutions $x^{0}(t)$ and $x^{1}(t)$ satisfying $x^{i}(0)=x^{i}$ such that $x^{i}(t) \in \mathcal{N}$ $\forall t \in\left[t_{k}, t_{k+1}\left[, k \geq 0\right.\right.$ and $h\left(x^{0}(t)\right) \neq h\left(x^{1}(t)\right)$ for some $t \in\left[t_{k}, t_{k+1}[\right.$, $k \geq 0$.

REMARK 2 The subsystem (9) given in $\boldsymbol{O C F}$ is $\boldsymbol{U}$-observable with respect to $Z(k)$, but the original subsystem (1) needs the knowledge of $Z(k)$ and $\Phi_{Z}^{-1}(\zeta)$ to be able to estimate $x$.

We denote by $\mathcal{I}\left(x^{0} ; U \subseteq \mathcal{N}\right)$ the set of all points $x^{1} \in U$,an open neighborhood of $x^{0}$, that are not uniformly distinguishable from $x^{0}$.

Definition 2 The system (1) is uniformly observable at $x^{0} \in N$ if $\mathcal{I}\left(x^{0} ; \mathcal{N}\right)=$ $x^{0}$ and is (Locally Uniformly) $\boldsymbol{L} \boldsymbol{U}$-observable at $x^{0} \in \mathcal{N}$ if, there exist an open neighborhood $U$ of $x^{0}$, such that $\mathcal{I}\left(x^{0} ; U\right)=x^{0}$.

Definition 3 The dynamics (1) is (Locally Regularly Weakly Uniformly) ${ }^{1}$ $\boldsymbol{L} \boldsymbol{R} \boldsymbol{W} \boldsymbol{U}$-observable ${ }^{2}$ at $x^{0}$, for all $t \in\left[t_{k}, t_{k+1}[\forall k>0\right.$, and $Z(k)$ is constant during the same interval if there exist $U\left(x^{0}\right)$, a neighborhood of $x^{0}$ and $p$ integers $\left(k_{1}, \ldots, k_{p}\right)$, independent from $Z(k)$ that form, after a possible reordering of the outputs, the smallest $p$-tuple with respect to the lexicographic ordering, such that for all $x \in U\left(x^{0}\right)$ and $Z(k)$ constant for $t \in\left[t_{k}, t_{k+1}[\right.$ $\forall k \geq 0$.

i) $k_{1} \geq k_{2} \geq \ldots \geq k_{p} \geq 0$

ii) $\sum_{i}^{p} k_{i}=n,\left(k_{i}\right.$ are observability indices of (1))

\footnotetext{
${ }^{1}$ The LRWU-observability leads to the LU-observability.

${ }^{2}$ This is a modified version of observability conditions given in 15 .
} 
iii) The rank observability condition is verified $\forall Z(k)$ and $x(t)$ with $t \in$ $\left[t_{k}, t_{k+1}[, k \geq 0\right.$

$$
\operatorname{rank}\left(\begin{array}{c}
d h_{1} \\
d L_{F} h_{1} \\
\vdots \\
d L_{F}^{\left(k_{1}-1\right)} h_{1} \\
\vdots \\
d h_{p} \\
\vdots \\
d L_{F}^{\left(k_{p}-1\right)} h_{p}
\end{array}\right)_{\mid x(t)}=n
$$

REMARK 3 The choice of $k_{i}$ is not unique and the assumption that the observability indices $k_{i}$ are not affected by $Z(k)$ is a strong one.

Under condition (5), (11)-(3) may be written in Observability Canonical Form (OCF) ([1] $), \forall t \in\left[t_{k}, t_{k+1}[\right.$ and $\forall k$ thanks to the diffeomorphism $\zeta=$ $\Phi_{Z}(x)$ defined as:

$$
\begin{aligned}
\zeta & =\left(\zeta_{1}^{T}, . ., \zeta_{i}^{T}, . ., \zeta_{p}^{T}\right)^{T} \\
& =\left[h_{1}(x), L_{F} h_{1}(x), \ldots, L_{F}^{k_{1}-1} h_{1}(x),\right. \\
& \left.\quad \ldots, h_{p}(x), L_{F} h_{p}(x), \ldots, L_{F}^{k_{p}-1} h_{p}(x)\right]^{T}
\end{aligned}
$$

with $\zeta_{i}=\left(\zeta_{1, i}, \zeta_{2, i}, \ldots, \zeta_{k_{i}, i}\right)^{T}$, for $i=1, . ., p$.

REMARK 4 Diffeomorphism $\Phi_{z(k)}(x)$ is parameterized by $Z(k)$ and is discontinuous at each switching instant $t_{k}$.

To guarantee the linearity of the $\mathbf{O C F}$, with respect to $Z(k)$, the following assumption is needed

Assumption 2 The continuous dynamics (1) should verify the following conditions :

i) $\exists$ at least $j \in\{1, \ldots, p\}$ such that

$$
\frac{\left.\partial\left(L_{F\left(x=\Phi_{Z}^{-1}(\zeta), Z\right)}^{k_{j}} h_{j}\right)\right|_{\zeta}}{\partial Z} \neq 0,
$$


ii) For all $i=1, . ., p$, we have

$$
\frac{\left.\partial^{2}\left(L_{F\left(x=\Phi_{Z}^{-1}(\zeta), Z\right)}^{k_{i}} h_{i}\right)\right|_{\zeta}}{\partial Z^{2}}=0
$$

Obviously, assumption 2 is sufficient for the considered problem and can be more relaxed with respect to the left invertibility problem ([6, 16]). Hence, subsystem (17)-(3), under conditions (5)-(7)-(8) and diffeomorphism (6) can be rewritten in $\mathbf{O C F}$ linear with respect to $Z(k)$ as:

$$
\begin{aligned}
& \left\{\begin{aligned}
\dot{\zeta}_{1, i} & =\zeta_{2, i} \\
\dot{\zeta}_{2, i} & =\zeta_{3, i} \\
& \vdots \\
\dot{\zeta}_{k_{i}, i} & =\left.\left(L_{F(x, Z)}^{k_{i}} h_{i}(x)\right)\right|_{x=\Phi_{Z}^{-1}(\zeta)} \\
& =\Gamma_{i}(\zeta)+\Lambda_{i}(\zeta) Z(k)=\Gamma_{i}(\zeta)+\sum_{j=1}^{m} \Lambda_{i j}(\zeta) z_{j}(k) \\
y_{i}= & \zeta_{1, i} \quad i=1, \ldots, p
\end{aligned}\right. \\
& \text { where }: \Gamma_{i}(\zeta)=\left.\left(L_{F(x, 0)}^{k_{i}} h_{i}\right)\right|_{x=\Phi_{0}^{-1}(\zeta)} i=1, . . p \\
& \Lambda_{i, j}(\zeta)=\frac{\left.\partial\left(L_{F\left(x=\Phi_{Z}^{-1}(\zeta), Z\right)}^{k_{i}} h_{i}\right)\right|_{\zeta}}{\partial z_{j}}, \quad j=1, . . m
\end{aligned}
$$

We note that the system (9) can present jumps even if it ont the case for the original one. In fact,

$$
x\left(t_{k}^{+}\right)=x\left(t_{k}^{-}\right)=x\left(t_{k}\right)
$$

using (6), for $t \in\left[t_{k-1}, t_{k}\left[\right.\right.$ and $t \in\left[t_{k}, t_{k+1}[\right.$, one has

$$
\begin{aligned}
& \zeta(t)=\Phi_{z(k-1)}(x(t)) \quad \forall t \in\left[t_{k-1}, t_{k}[\right. \\
& \zeta(t)=\Phi_{z(k)}(x(t)) \quad \forall t \in\left[t_{k}, t_{k+1}[\right.
\end{aligned}
$$

Now, starting from these equations (11)-(12) and (10), at the sampling instants $t_{k}$ one obtains:

$$
\zeta\left(t_{k}^{+}\right)=\Phi_{z(k)}\left[\Phi_{z(k-1)}^{-1}\left(\zeta\left(t_{k}^{-}\right)\right)\right]=\mathcal{R}_{[z(k-1), z(k)]}\left(\zeta\left(t_{k}^{-}\right)\right)
$$


This relation gives the reset equation of $\zeta(t)$ at time $t_{k}^{+}$just after the switching time.

The following proposition summarize the previous result :

Proposition 1 Under conditions (5)-(7)-(8), the subsystem (1) is transformable by diffeomorphism parametrized by $Z(k)$ into the $\boldsymbol{O C F}$ (9).

REMARK 5 As the subsystem (9) is given in $\boldsymbol{O C F}$, it is $\boldsymbol{U}$-observable with respect to $Z(k)$ ([1A]). Nevertheless, in order to reconstruct the original state $x$ of (1), the knowledge of $\Phi_{Z}^{-1}(\zeta)$ is necessary and consequently $Z(k)$ $\forall k$.

To overcome this difficulty, we will use a software sensor from the continuous dynamics (written in $\mathbf{O C F}$ ), in order to obtain information with respect to the discrete states $Z(k)$.

Thus, from (9), all the last row of the sub-dynamics of $\zeta_{i}\left(\zeta_{k_{i}, i}\right.$ for $i=$ $1, . ., p)$, may be written as:

$$
\dot{\theta}=\Gamma(\zeta)+\Lambda(\zeta) Z(k)
$$

with $\theta=\left[\zeta_{k_{1}, 1,}, \ldots, \zeta_{k_{p}, p}\right]^{T}$, and $\Gamma(\zeta)=\left[\Gamma_{1}(\zeta), \ldots, \Gamma_{p}(\zeta)\right]^{T}$ is a column vector of dimension $p$, and

$$
\left.\Lambda(\zeta)\right|_{x=\Phi_{Z}^{-1}(\zeta)}=\left[\Lambda_{1}^{T}(\zeta), \ldots, \Lambda_{m}^{T}(\zeta)\right]^{T}
$$

is a $p \times m$ matrix and $\Lambda_{i}(\zeta)$ is a line vector of dimension $m$ defined as:

$$
\Lambda_{i}(\zeta)=\left[\Lambda_{i, 1}(\zeta), \ldots, \Lambda_{i, m}(\zeta)\right], \quad i=1, \ldots, p
$$

For the sake of simplicity, and because the continuous states in $\zeta$ coordinates are also known after the convergence of the continuous observer, we choose to consider each $\Lambda_{i, j}(\zeta)$ at time $t_{k}^{-}$, i.e., $\Lambda_{i, j}\left(\zeta\left(t_{k}\right)\right)=\Lambda_{i, j}\left(\zeta\left(t_{k}^{-}\right)\right)$, to guarantee the finite time convergence of the continuous observer.

The discrete dynamics (2) remains linear in $Z(k)$, and will be completed by an output $Y_{d}(k)=\Lambda(\zeta(k)) Z(k)$ :

$$
\begin{aligned}
Z(k+1) & =A_{d} Z(k)+\beta_{d}(v(k), y(k)) \\
Y_{d}(k) & =\Lambda(\zeta(k)) Z(k)
\end{aligned}
$$

Where $\Lambda(\zeta(k))=\Lambda\left(\zeta\left(t=t_{k}^{-}\right)\right)$.

The discrete subsystem (15)-(16) will now be used to design a discrete observer. As $\Lambda(\zeta(k))$ is a $(p \times m)$ constant matrix only at time $t \in\left[t_{k}, t_{k+1}[\right.$ but may change at each $t_{k} \forall k>0$, we need to define the distinguishability and observability of (15)-(16). 
Definition $4 A$ pair of points $Z^{0}$ and $Z^{1}$ in $\mathcal{M}$ is uniformly distinguishable with respect to $v(k) \forall k>0$, and $\forall \zeta(0)$ if the system (15)-(10) generates, $\forall \zeta(0)$ and $v(k)$, solutions $Z^{0}(k)$ and $Z^{1}(k)$ satisfying $Z^{i}(0)=Z^{i}$ with $Z^{i}(k) \in \mathcal{M}$, and there exists $k$ such that $\Lambda(\zeta(k)) Z^{0}(k) \neq \Lambda(\zeta(k)) Z^{1}(k)$.

We denote by $\mathcal{J}\left(Z^{0} ; \mathcal{M}\right)$ all points $Z^{1} \in \mathcal{M}$ that are not uniformly distinguishable from $Z^{0}$.

Definition 5 The system (15)-(10) is uniformly observable at $Z^{0} \in \mathcal{M}$ if $\mathcal{J}\left(Z^{0} ; \mathcal{M}\right)=\left\{Z^{0}\right\}$.

Definition 6 The system (15)-(10) is, in a delayed way, $\boldsymbol{U}$-observable at $Z^{0} \in \mathcal{M}$, if there exist a bounded $\mu \geq 0$ such that, for all $\zeta(0)$ and $v(k)$, $\forall k<\mu$ the system is uniformly observable at $Z(\mu)$.

The above definition means that, even if the reconstruction of $Z(0)$ is not guaranteed, then the reconstruction of $Z(\mu)$ may be possible. In fact, to reconstruct a state vector $Z$ at time $\mu$ starting from equation (15)-(16) with the following notations $Y_{d}^{\mu}:=Y_{d}(\mu), \zeta_{\mu}:=\zeta(\mu), Z_{\mu}:=Z(\mu)$, and $\beta_{d, \mu}:=\beta_{d}(v(\mu), y(\mu))$, one obtains:

$$
\begin{aligned}
Y_{d}^{\mu}= & \Lambda\left(\zeta_{\mu}\right) Z_{\mu} \\
Y_{d}^{\mu+1}= & \Lambda\left(\zeta_{\mu+1}\right)\left[A_{d} Z_{\mu}+\beta_{d, \mu}\right] \\
& \vdots \\
Y_{d}^{\mu+N}= & \Lambda\left(\zeta_{\mu+N}\right) A_{d}^{N} Z_{\mu}+\Lambda\left(\zeta_{\mu+N}\right) \sum_{i=1}^{N} A_{d}^{N-i} \beta_{d, \mu+i-1}
\end{aligned}
$$

which can be rewritten as:

$$
\left[\begin{array}{c}
Y_{d}^{\mu} \\
Y_{d}^{\mu+1} \\
\vdots \\
Y_{d}^{\mu+N}
\end{array}\right]=\left[\begin{array}{c}
\Lambda\left(\zeta_{\mu}\right) \\
\Lambda\left(\zeta_{\mu+1}\right) A_{d} \\
\vdots \\
\Lambda\left(\zeta_{\mu+N}\right) A_{d}^{N}
\end{array}\right] Z_{\mu}+\left[\begin{array}{c}
D_{\mu} \\
D_{\mu+1} \\
\vdots \\
D_{\mu+N}
\end{array}\right]
$$

where $D_{\mu+j}=\Lambda\left(\zeta_{\mu+j}\right) \sum_{i=1}^{j} A_{d}^{j-i} \beta_{d, \mu+i-1}$ for $j=1, . ., N$, is a known expression depending only on $(v(),. y()$.$) .$

Proposition 2 (Observability of $\boldsymbol{Z}(\boldsymbol{\mu})$ ) The discrete system (15)-(10) is observable at $Z(\mu) \in \mathcal{M}$ for a fixed $\zeta(\mu) \in \mathcal{N}$ and for a fixed set of input 
$v(k), k>\mu$ if and only if $\left(\Lambda(),. A_{d}\right)$, verifies the following observability conditions:

There exists a bounded integer $\tilde{N}$ such that:

$$
\operatorname{rank}\left\{\bar{\Lambda}_{\mu}=\left(\left[\begin{array}{c}
\Lambda\left(\zeta_{\mu}\right) \\
\Lambda\left(\zeta_{\mu+1}\right) A_{d} \\
\vdots \\
\Lambda\left(\zeta_{\mu+\tilde{N}}\right) A_{d}^{\tilde{N}}
\end{array}\right]\right)\right\}=m
$$

\section{Proof.}

- Sufficiency: From (17), verifying (18) and using the Left Pseudo Inverse, one obtains $Z(k)$.

- Necessary: If for all integer $\tilde{N}, \operatorname{rank}\left\{\bar{\Lambda}_{\mu}\right\}<m$, then $\operatorname{Ker}\left\{\bar{\Lambda}_{\mu}\right\}$ is at least of dimension 1 , and consequently the unobservable space is at least of dimension one.

- The construction of the matrix $\bar{\Lambda}_{\mu}$ is based on FIFO (First Input First Output) principle.

COROLlaRY 1 (Uniform Observability of $\boldsymbol{Z}(\boldsymbol{\mu})$ ) The discrete system (15)-(10) is $\boldsymbol{U}$-observable at $Z(\mu) \in \mathcal{M}, \forall \zeta(0) \in U$ and $\forall v(k)$ for $k>\mu$, if and only if $\left(\Lambda(),. A_{d}\right)$, verifies the following observability conditions:

There exists a bounded integer $N(\zeta(0), v(k))$, noted $N$ such that:

$$
\operatorname{rank}\left\{\bar{\Lambda}_{\mu}=\left(\left[\begin{array}{c}
\Lambda\left(\zeta_{\mu}\right) \\
\Lambda\left(\zeta_{\mu+1}\right) A_{d} \\
\vdots \\
\Lambda\left(\zeta_{\mu+N}\right) A_{d}^{N}
\end{array}\right]\right)\right\}=m
$$

REMARK 6 If $\mu=0$, the previous corollary leads to the $\boldsymbol{U}$-Observability

Obviously, for causality reasons, $\mu+N$ is the present instant usually noted $k$ and we don't know $Z$ at time $k$ but $k-N$ and then equation (17) will be rewritten as

$$
\left[\begin{array}{c}
Y_{d}^{k-N} \\
Y_{d}^{k-N+1} \\
\vdots \\
Y_{d}^{k}
\end{array}\right]=\left[\begin{array}{c}
\Lambda\left(\zeta_{k-N}\right) \\
\Lambda\left(\zeta_{k-N+1}\right) A_{d} \\
\vdots \\
\Lambda\left(\zeta_{k}\right) A_{d}^{N}
\end{array}\right] Z_{k-N}+\left[\begin{array}{c}
D_{k-N} \\
D_{k-N+1} \\
\vdots \\
D_{k}
\end{array}\right]
$$

Now, from definitions (2)-(3) and (5)-(6), we are able to propose an observability definition for the whole HDS system defined in (11). 
Definition 7 A pair of continuous and discrete state $\left(x^{0}(0), z^{0}(0)\right)$ and $\left(x^{1}(0), z^{1}(0)\right)$ in $\mathcal{N} \times \mathcal{M}$ are uniformly distinguishable if for all input $v(k)$, the system (1) generates solutions $\left(x^{0}(t), z^{0}(t)\right)$ and $\left(x^{1}(1), z^{1}(1)\right)$ such that $h\left(x^{0}(t)\right) \neq h\left(x^{1}(t)\right)$ for some $t \neq+\infty$.

We denote by $£\left(x^{0}, z^{0} ; U \times \mathcal{M}\right)$ all sets of couple $\left(x^{1}, z^{1}\right) \in U \times \mathcal{M}$ that are not distinguishable from $\left(x^{0}, z^{0}\right)$.

Definition 8 The class of HDS (1) is (Locally Uniformly) $\boldsymbol{L} \boldsymbol{U}$-observable at $\left(x^{0}, z^{0}\right) \in \mathcal{N} \times \mathcal{M}$ if $£\left(x^{0}, z^{0} ; U \times \mathcal{M}\right)=\left(x^{0}, z^{0}\right)$.

DEFINITION 9 The hybrid system (四) is in delayed way (Locally Uniformly) $\boldsymbol{L} \boldsymbol{U}$-Observable at $\left(x^{0}, Z^{0}\right)$ if there exists a bounded integer $k>0$, such that, the system (1) is $\boldsymbol{L} \boldsymbol{U}$-Observable at $(x(k), Z(k))$

Proposition 3 If the continuous dynamics (1) verify conditions (5)-(17)(8) and it is (Locally Regularly Weakly) $\mathbf{L} \boldsymbol{R} \boldsymbol{W}$-Observable at $x^{0}$, for all $t \in\left[t_{k}, t_{k+1}[\forall k\right.$ and the subsystems (15)-(10) is uniformly observable at $z^{0} \in M$, then the whole HDS defined in (1) is (Locally Uniformly) $\boldsymbol{L} \boldsymbol{U}$ observable at $\left(x^{0}, z^{0}\right)$ and it is $\boldsymbol{L} \boldsymbol{U}$-observable, in delayed way, at $\left(x^{t_{\mu}}, z^{\mu}\right)$ for $\mu>0$, if the subsystems (15)-(16) is uniformly observable in delayed way at $z^{\mu} \in \mathcal{M}$.

In the next, a high order sliding modes observer combined with discrete state reconstructor will be designed for this class of HDS which is LU-Observable (or in a delayed way LU-Observable). Advantages of such observer, with respect to other ones which may be used, are its finite time convergence, and its ability to consider the variable structure systems.

\section{Hybrid Observer Design}

The hybrid observer design is carried out in the following way : (figure 1). Once the diffeomorphism carried out, a sliding mode based observer (Super Twisting Algorithm), is firstly applied to the continuous system transformed in the OCF (9). Secondly, after the estimation of all continuous states of the system in the $\zeta$ coordinates in $n-1$ first steps, the last $n^{\text {th }}$ step allows reconstructing the whole dynamics and by consequence obtaining $Y_{d}(k)=\Lambda\left(\tilde{\zeta}\left(t_{k}^{-}\right)\right) Z(k)=\Lambda\left(\tilde{\zeta}\left(k^{-}\right)\right) Z(k)$. The discrete observer consists in using $Y_{d}(k)$ like an output for the discrete dynamics, in order to design a discrete observer to estimate $\hat{Z}(k)$. 


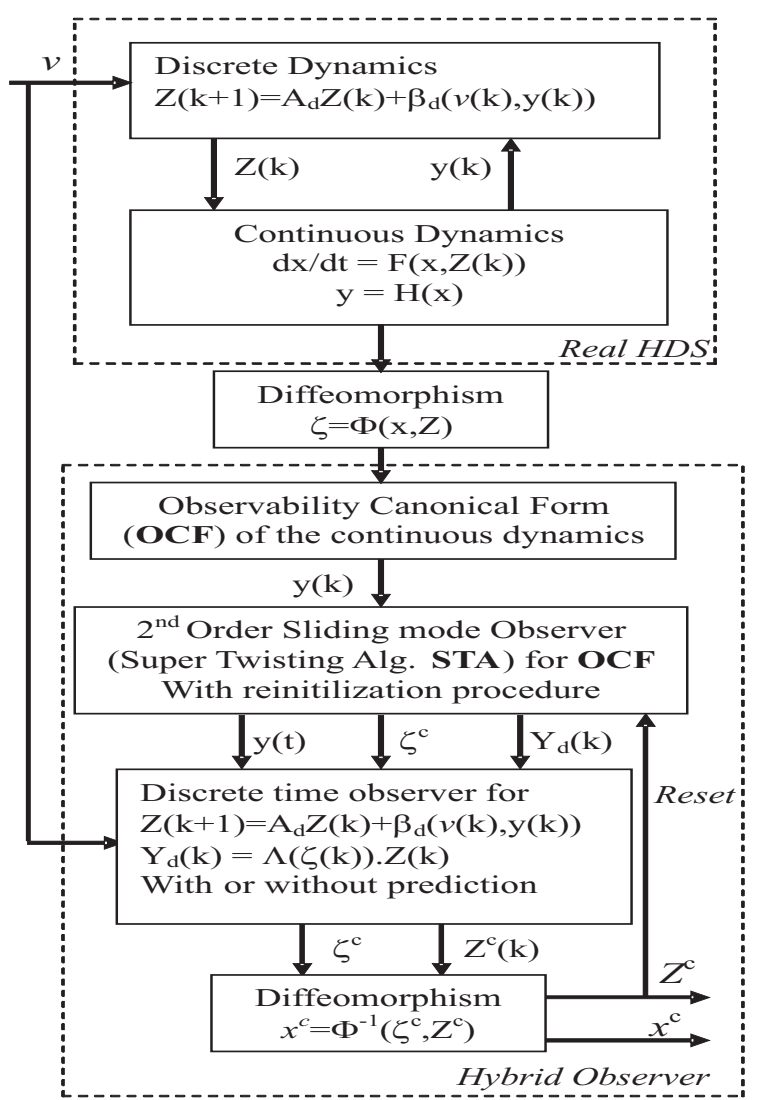

Fig. 1 : Structure of the Hybrid Observer

Continuous and Discrete States with : $Z^{c}=\hat{Z}, \zeta^{c}=\hat{\zeta}$ et $x^{c}=\hat{x}$ 


\subsection{Super Twisting Algorithm}

In [20], a Super Twisting Algorithm (STA) was used for switched system. In our case, a STA is used for the continuous part of the system. In fact, the continuous dynamics will be supposed as a forced commutation, since it is the discrete dynamics which makes the difference while $Z(k)$ change its value.

The STA is given by the following structure 17]:

$$
\begin{aligned}
\sum_{o b s}= & \left\{\begin{array}{l}
u\left(e_{1}\right)=u_{1}+\lambda_{1}\left|e_{1}\right|^{\frac{1}{2}} \operatorname{sign}\left(e_{1}\right) \\
\dot{u}_{1}=\alpha_{1} \operatorname{sign}\left(e_{1}\right)
\end{array}\right. \\
& \lambda_{1}, \alpha_{1}>0
\end{aligned}
$$

where $e_{1}=\zeta_{1, i}-\hat{\zeta}_{1, i}, \lambda_{1}, \alpha_{1}$ are positive parameters, and $u_{1}$ is the differentiator output where:

$$
\operatorname{sign}(.)=\left\{\begin{array}{c}
+1 \text { if }(.)>0 \\
-1 \text { if }(.)<0 \\
\in[-1,1] \text { if }(.)=0
\end{array}\right.
$$

The step by step exact differentiator applied to each subsystem (9), leads to the following form:

$$
\begin{aligned}
& \dot{\hat{\zeta}}_{1, i}=\tilde{\zeta}_{2, i}+\lambda_{1, i}\left|\tilde{e}_{1, i}\right|^{1 / 2} \operatorname{sign}\left(\tilde{e}_{1, i}\right) \\
& \dot{\tilde{\zeta}}_{2, i}=\alpha_{1, i} \operatorname{sign}\left(e_{1, i}\right) \\
& \dot{\hat{\zeta}}_{2, i}=E_{1, i}\left[\tilde{\zeta}_{3, i}+\lambda_{2, i}\left|\tilde{e}_{2, i}\right|^{1 / 2} \operatorname{sign}\left(\tilde{e}_{2, i}\right)\right] \\
& \vdots \\
& \dot{\hat{\zeta}}_{k_{p}-1, i}=E_{k_{p}-2, i}\left[\tilde{\zeta}_{k_{p}, i}+\ldots\right. \\
& \left.\quad+\lambda_{k_{p}-1, i}\left|\tilde{e}_{k_{p}-1, i}\right|^{1 / 2} \operatorname{sign}\left(\tilde{e}_{k_{p}-1, i}\right)\right] \\
& \dot{\tilde{\zeta}}_{k_{p}, i}=E_{k_{p}-2, i} \alpha_{k_{p}-1, i} \operatorname{sign}\left(\tilde{e}_{k_{p}-1, i}\right) \\
& \dot{\hat{\zeta}}_{k_{p}, i}=E_{k_{p}-1, i}\left[\tilde{\theta}_{i}+\lambda_{k_{p}, i}\left|\tilde{e}_{k_{p}, i}\right|^{1 / 2} \operatorname{sign}\left(\tilde{e}_{k_{p}, i}\right)\right] \\
& \dot{\tilde{\theta}}_{i}=E_{k_{p}-1, i} \alpha_{k_{p}, i} \operatorname{sign}\left(\tilde{e}_{k_{p}, i}\right)
\end{aligned}
$$

for $i=1, \ldots, p$, where $\tilde{e}_{j, i}=\tilde{\zeta}_{j, i}-\hat{\zeta}_{j, i}$, with $\tilde{\zeta}_{1, i}=\zeta_{1, i}$ for $j=1, . ., k_{p}$, and the vector $\tilde{\theta}_{i}$ represent the estimation of the dynamic $\Gamma_{i}(\zeta)+\Lambda_{i}(\zeta) Z(k)$ and $E_{j, i}$ for $j=1, \ldots k_{i}-1$ are defined as

$$
E_{j, i}=0 \text { if } \quad\left|\tilde{e}_{j, i}\right| \geq \varepsilon>0, \quad \text { else } \quad E_{j, i}=1
$$


See 13 for more details concerning the convergence proof and the definition of $E_{j, i}$. We also have to take into account the jumps (13) due to the diffeomorphism and use the reinitialisation procedure $\forall t=t_{k}, k>0$.

$$
\hat{\zeta}^{+}\left(t_{k}\right)=\Phi_{\hat{z}(k)}\left[\Phi_{\hat{z}(k-1)}^{-1}\left(\hat{\zeta}\left(t_{k}^{-}\right)\right)\right]
$$

The convergence of the observation error for all the continuous states is obtained in $(n-1)$ steps in finite time and allows to reconstruct the state vector. To obtain the discrete output $Y_{d}=\Lambda(\tilde{\zeta}) Z(k)$, let us consider:

$$
\tilde{\theta}(k)=\Gamma\left(\tilde{\zeta}\left(k^{-}\right)\right)+\Lambda\left(\tilde{\zeta}\left(k^{-}\right)\right) Z(k)
$$

where $k^{-}$is the time $t=t_{k}^{-}$(just before switching instant) then at time $t=t_{k}$ one obtains:

$$
Y_{d}(k)=\Lambda\left(\tilde{\zeta}\left(k^{-}\right)\right) Z(k)=\left[\tilde{\theta}(k)-\Gamma\left(\tilde{\zeta}\left(k^{-}\right)\right)\right]
$$

Thanks to the canonical form, the observation of $\zeta$ doesn't need the knowledge of $Z(k)$. In fact, the sliding mode observer based on the STA needs only to know $y_{i}=\zeta_{i, 1}$. This is why the hybrid observer design is carried out in two sccessives stages, starting with a continuous observer. The hybrid observer design requires the following condition:

CONDITION 1 The sliding mode observer is designed such that the convergence time $\tau_{c}^{k}$ during the interval $\left[t_{k}, t_{k+1}[\forall k \geq 0\right.$ verifies the following condition:

$$
\tau_{m}>\max \left\{\tau_{c}^{k}\right\}>0 \quad \forall k \geq 0
$$

with $\tau_{m}$ is defined in (4).

Condition (बi) is justified by the fact that the convergence time $\tau_{c}^{k}$ of the designed observer for each constant value of $Z(k)$ is not the same and also ensures that the designed observer has the time to estimate the continuous state during $\left[t_{k}, t_{k+1}[\forall k \geq 0\right.$. The following proposition summarizes the properties of the observer (22) (see [13] for more details).

Proposition 4 Let us consider the system (9), supposed to be bounded for all $t \in\left[t_{k}, t_{k+1}[\forall k>0\right.$, and a sliding mode observer (29). For any initial condition $\zeta(k), \hat{\zeta}(k)$, and for all $Z(k)$, there exist bounded $\alpha_{i, j}$ and $\lambda_{i, j}$ such that $\hat{\zeta}$ converges in finite time (smaller than $\tau_{m}$ ) towards $\zeta$, i.e. $\exists$ $t=\tau_{c}^{k}>0$ such that $\tilde{\zeta}=\zeta$, and $\tilde{\theta}$ converges towards $\Gamma(\tilde{\zeta})+\Lambda(\tilde{\zeta}) Z(k)$, and by consequence reconstruct a discrete output $Y_{d}(k)=\Lambda(\tilde{\zeta}) Z(k)$ as in (25).

All switching instants are known by both observers and the reset information (24) is transmitted from the discrete observer to the continuous one. 


\subsection{Discrete state observer}

In this section, we will design the observer of the discrete subsystem to estimate the discrete state. Let us consider the system (1) and the observation structure given in the previous section, the discrete observer receives as input : the observed state $\tilde{\zeta}$, the output $y(k)$, the information $E_{k_{i}-1, i}$ for $i=1, . ., p$ and $v(k)$. Its task is to provide an estimation $\hat{Z}$ of the discrete state $Z$ of the hybrid plant starting from both the reconstructed discrete output $Y_{d}(k)$ given in (25). and the sampled continuous state $\tilde{\zeta}(k)$. To design the discrete observer, let us consider that (1) verifies the observability condition (18). After the estimation of the continuous state $\tilde{\zeta}$ and a discrete output $Y_{d}=\Lambda(\tilde{\zeta}(k)) Z(k)$, the following system will be used to estimate $\hat{Z}$ :

$$
\begin{aligned}
& Z(k+1)=A Z(k)+\beta_{d}(v(k), y(k)) \\
& Y_{d}=\Lambda(\tilde{\zeta}(k)) Z
\end{aligned}
$$

If condition (18) is verified, it is possible to design an observer for the time varying system (27) to obtain $\hat{Z}(k)$ which converges to $Z(k)$. Note that the designed method performed in [2, 18], for finite time convergent observers for linear time-varying systems, can be used in our case.

The estimation $\hat{Z}(k-N)$ can also be obtained by using left invertibility on the equation (20), one obtains:

$$
\hat{Z}_{k-N}=\left[\begin{array}{c}
\Lambda\left(\zeta_{k-N}\right) \\
\vdots \\
\Lambda\left(\zeta_{k}\right) A_{d}^{N}
\end{array}\right]^{-1 *}\left(\begin{array}{l}
Y_{d}^{k-N}-D_{k-N} \\
\vdots \\
Y_{d}^{k}-D_{k}
\end{array}\right)
$$

where $(.)^{-1 *}$ represents a pseudo inverse of (.).

Obviously, as it is mentioned before, for causality reasons and in order to estimate the discrete state at time $k$ a discrete time predictor of $N$-steps will be used as follows:

Starting from (27) and denoting $\beta_{d, k-N}=\beta_{d}(v(k-N), y(k-N))$, and $Z_{k}=Z(k)$, one obtains:

$$
\begin{aligned}
\hat{Z}_{k-N+1}= & A_{d} \hat{Z}_{k-N}+\beta_{d, k-N} \\
\hat{Z}_{k-N+2}= & A_{d}^{2} \hat{Z}_{k-N}+A_{d} \beta_{d, k-N+1}+\beta_{d, k-N} \\
& \vdots \\
\hat{Z}_{k}= & A_{d}^{N} \hat{Z}_{k-N}+\sum_{i=1}^{N} A_{d}^{N-i} \beta_{d, k-N+i-1}
\end{aligned}
$$

which gives the prediction of the discrete state $Z$ at time $k$ starting from the knowledge of $Z(k-N)$. 


\section{Illustrative example}

where $x=\left(x_{1}, x_{2}, x_{3}, x_{4}\right)^{T}, Z=\left(z_{1}, z_{2}, z_{3}\right)^{T}, y_{1}=x_{1}, y_{2}=x_{3}$

$$
F(x, Z(k))=\left(\begin{array}{l}
x_{2}+z_{1}(k) \\
-10 x_{2}-5 \sin \left(0.5 x_{3}\right)-x_{1}^{2} z_{1}(k) \\
x_{4}+z_{1}(k) \\
-x_{3}^{2}+5 x_{1}+x_{1} z_{2}(k)
\end{array}\right)
$$

and

$$
A_{d}=\left(\begin{array}{ccc}
0.50 & 0 & 1 \\
-0.2 & 0.1 & 0 \\
-0.20 & 0 & -0.1
\end{array}\right), \beta_{d}=\left(\begin{array}{c}
y_{1}(k) \\
0 \\
y_{2}(k)
\end{array}\right)
$$

Using the following diffeomorphism $\zeta=\Phi_{Z}(x)$ as $\zeta_{1}=x_{1} ; \zeta_{2}=x_{2}+z_{1}(k)$; $\zeta_{3}=x_{3}$ and $\zeta_{4}=x_{4}+z_{1}(k)$, one obtains

$$
\begin{aligned}
& \dot{\zeta}_{1}=\zeta_{2} \\
& \dot{\zeta}_{2}=-10 \zeta_{2}-5 \sin \left(0.5 \zeta_{3}\right)-\zeta_{1}^{2} z_{1}(k)+10 z_{1}(k) \\
& \dot{\zeta}_{3}=\zeta_{4} ; \\
& \dot{\zeta}_{4}=-\zeta_{3}^{2}+5 \zeta_{1}+\zeta_{1} z_{2}(k)-z_{1}(k) \\
& Z(k+1)=A_{d} Z(k)+\beta_{d}(y(k)) \\
& y_{1}=\zeta_{1} ; \quad \text { and } \quad y_{2}=\zeta_{3}
\end{aligned}
$$

with $Z(t)=Z(k)$ for all $t \in[T k, T(k+1)[$. To simplify the study, we consider a constant sampling period $T=t_{k+1}-t_{k}=0.5 \mathrm{~s}$. The associated continuous observer is then given as in (22):

After a finite time, one obtains $\hat{\zeta}$ and then $Y_{d}=\Lambda(\zeta) Z(k)$ in the following way:

$$
\begin{aligned}
-\zeta_{1}^{2}(k) \tilde{z}_{1}(k) & =\left(\tilde{\theta}_{1}(k)-10 \zeta_{2}(k)-0.5 \sin \left(\zeta_{3}(k)\right)\right) \\
\zeta_{1}(k) \tilde{z}_{2}(k) & =\left(\tilde{\theta}_{2}(k)+\zeta_{3}^{2}(k)-5 \zeta_{1}(k)\right)
\end{aligned}
$$

and thus, if the output $Y_{d}(k)$ is considered, one can use a pseudo inverse or left inversion to obtain $\hat{Z}(k)$.

using (24), the reinitialization procedure is given by

$$
\begin{array}{ll}
\hat{\zeta}_{1}^{+}\left(t_{k}\right)=\hat{\zeta}_{1}\left(t_{k}^{-}\right) ; & \hat{\zeta}_{2}^{+}\left(t_{k}\right)=\hat{\zeta}_{2}\left(t_{k}^{-}\right)-z_{1}(k-1)+z_{1}(k) \\
\hat{\zeta}_{3}^{+}\left(t_{k}\right)=\hat{\zeta}_{3}\left(t_{k}^{-}\right) ; & \hat{\zeta}_{4}^{+}\left(t_{k}\right)=\hat{\zeta}_{4}\left(t_{k}^{-}\right)-z_{1}(k-1)+z_{1}(k)
\end{array}
$$

Now, $\hat{Z}(k-1)$ is given by equation (28) and to obtain $\hat{Z}(k)$, one uses (29) to realize the predictor with $N=1$ :

$$
\hat{Z}_{k}=A_{d} \hat{Z}_{k-1}+\beta_{d, k-1}
$$


Finally, using $\Phi_{Z}^{-1}$, one obtains the estimation of the continuous state in the original coordinates. The simulation results are depicted in (figures 2 Q 4 )
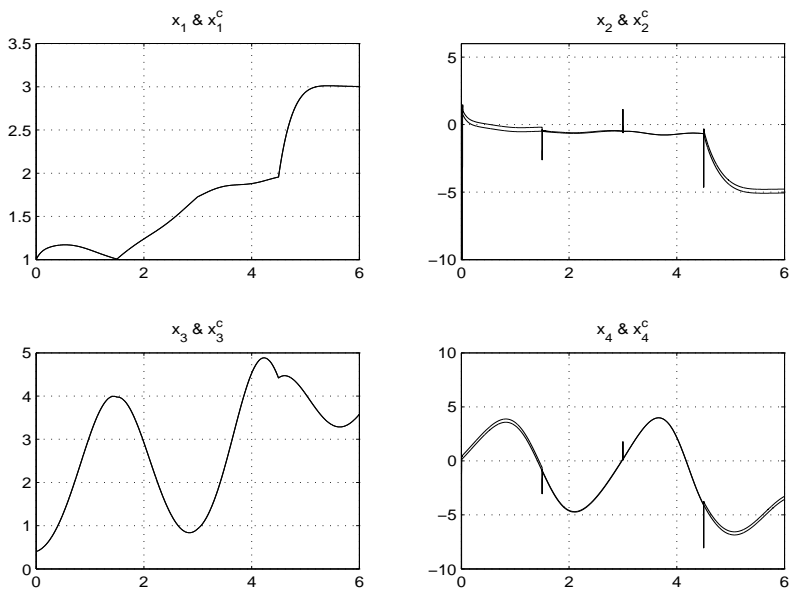

Fig. 2 : Real $x$ and observed $\hat{x}$ continuous states
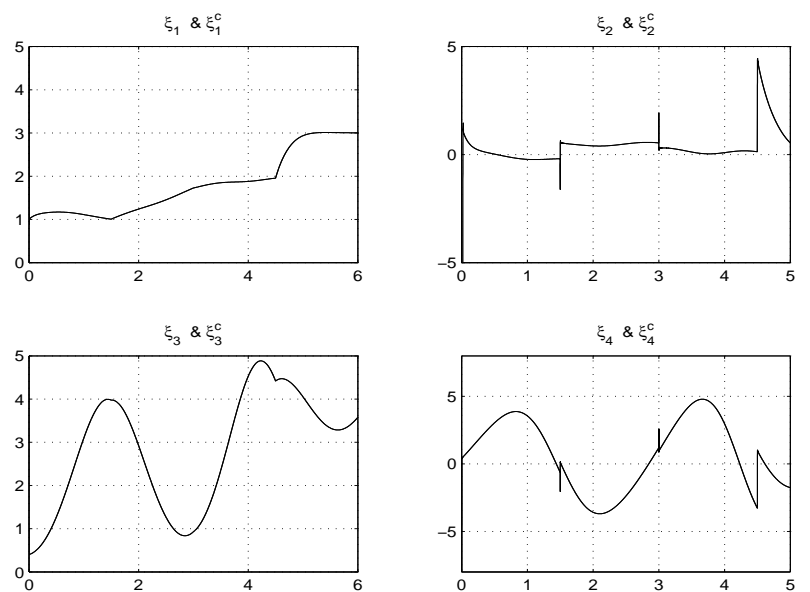

Fig. 3 : Real $\xi$ and observed $\hat{\xi}$ continuous states

\section{References}

[1] Antsaklis, P.J., editors (2000). Proceedings of the IEEE, Special Issue on Hybrid Systems: Theory and Applications, 43(2). 

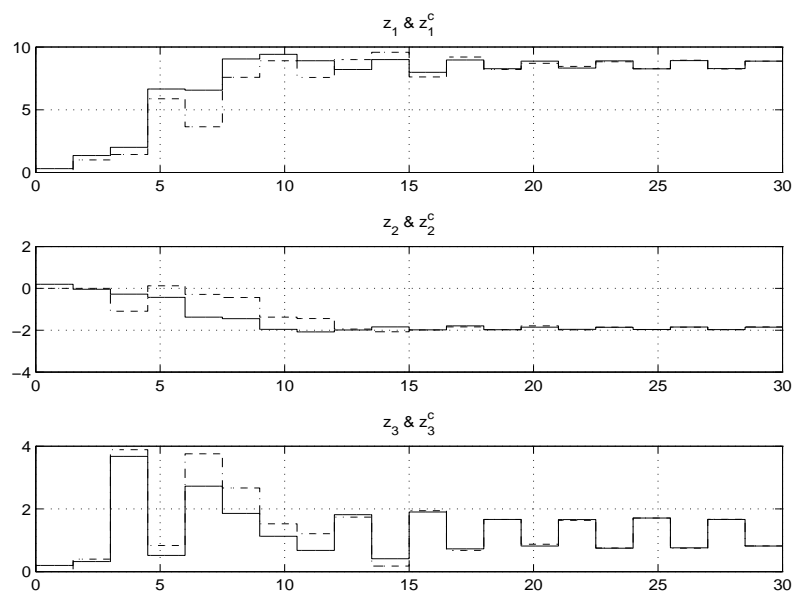

Fig. 4 : Real and observed discrete states

[2] Babaali, M., M. Egerstedt, and E. W. Kamen. (2003). An Observer for Linear Systems with Randomly-Switching Measurement Equations In Proc. of the American Control Conf. Denver, Colorado June 4-6, 2003

[3] Baglietto, M., G. Battistelli and L. Scardovi. (2006). Active mode observability of switched linear systems, In Proc. $45^{\text {th }}$ IEEE-CDC, San Diego, USA. pp. 145150 .

[4] Balluchi, A., L. Benvenuti, M.D. Di Benedetto and A.L. Sangiovanni Vincentelliy. (2001). A Hybrid Observer for the Driveline Dynamics. ECC, Porto, Portugal

[5] A. Balluchi and L. Benvenuti, M.D. Di Benedetto and A.L. SangiovanniVincentelli, (2003). Observability for Hybrid Systems, 42nd IEEE-CDC Maui, Hawaii USA, December 2003.

[6] Barbot, J.P., and T. Floquet. (2005). State and unknown input estimation for linear discrete-time systems", IFAC World Congress, Prague.

[7] Bemporad, A., G. Ferrari-Trecate and M. Morari. (2000). Observability and controllability of piecewise affine and hybrid systems. IEEE Trans on Automatic Control, 45 No. 10, pp. 1864-1876.

[8] De Santis, E., M.D. Di Benedetto and G. Pol a. (2006). Observability of internal variables in interconnected switching systems. In Proc. $45^{\text {th }}$ IEEE-CDC, pp. 4121-4126. 
[9] Djemai, M., and J. P. Barbot. (1995). Singularly Perturbed Method for the Control Design of a Synchronous Motor with its PWM Inverter. In proc. of IEEE-Conf. on Control Application, CCA'95, New York, USA.

[10] Djemai, M., N. Manamani and J. P. Barbot. (2005). Sliding Mode Observer For Triangular Input Hybrid System. IFAC World congres Prague, 2005.

[11] Fliess M. (1990). Generalized Controller Canonical Forms for Linear and Nonlinear Dynamics. IEEE Trans on Automatic Control, 35, No 9, pp. 994-1000.

[12] Fliess M., C. Join, M. Mboup, H. Sira-ramirez, (2005). Analyse et représentation de signaux transitoires : application a la compression, au débruitage et à la détection de ruptures. IEEE 20ème colloque sur le traitement du signal et de l'image GRETSI, Louvain-la-Neuve? Belgique.

[13] Floquet. T, and J.P. Barbot. (2007). Super twisting algorithm based step-bystep sliding mode observers for nonlinear systems with unknown inputs. In Int. J. of Control, To appear.

[14] Gauthier, J. P., and G. Bornanrd, (1981) Observability for any u(t) of a class of nonlinear systems, IEEE Trans on Automatic Control, 26, No 4, pp. 922-926.

[15] Hermann, R. and Krener, A.J., (1977), Nonlinear controllability and Observability, IEEE Trans on Automatic Control, 22, No 9, pp. 728-740.

[16] Hirschorn, R.M. (1979), Invertibilty of multivariable nonlinear control systems, IEEE Trans on Automatic Control, 24, pp. 855-865.

[17] Levant, A. (1998), Robust Exact Differentiation via sliding mode technique, In Automatica, Vol. 34, No. 3, pp. 379-384.

[18] Menold, P.H., R. Findeisen and F. Allgöwer. (2003). The peaking Finite time convergent observers for linear time-varying systems In Proc. of 42nd IEEECDC Conf. Maui, Hawaii USA, December 2003

[19] Petterson, S. (2003). Sythesis of switched Linear Systems, In Proc. $42^{\text {nd }}$ IEEECDC, Hawaii, USA.

[20] Saadaoui,H., N. Manamanni, M. Djemaï, J.P. Barbot and T. Floquet. (2005). Exact differentiation and Sliding mode observer for switched Lagrangian systems. in Nonlinear Analysis: Theory, Methods 83 Appl., Elsevier, pp. 10501069, (6). 
[21] Vidal, R., A. Chiuso, S. Soatto and S.S. Sastry. (2003). Observability of linear hybrid systems. In Hybrid Systems: Comput. and Cont., Lecture Notes in Computer Science, Springer Verlag, Vol. 2623, pp. 526-539.

[22] Vas, P., (1998). Sensorless Vector and Direct Torque Control (Monographs in Electrical and Electronic Engineering) OXFORD Science Publications. 